\title{
Complicaciones postoperatorias en esofagectomía por cáncer. Evaluación de 215 casos según definiciones del grupo de consenso internacional
}

\author{
Enrique Eduardo Norero M. ${ }^{1}$, Marco Ceroni V. ${ }^{1}$, Cristian Martínez B. ${ }^{1}$, \\ Ricardo Mejía M. ${ }^{1}$, Rodrigo Muñoz C. ${ }^{1}$, Constanza Godoy S. ${ }^{1}$, \\ Fernando Araos V. ${ }^{1}$, Paulina González C. ${ }^{1}$, Sergio Saldías C. ${ }^{1}$, \\ Marcelino Véliz L. ${ }^{1}$, Lucía Marinkovic R. ${ }^{1}$, Mario Caracc L. ${ }^{1}$, Alfonso Díaz F. ${ }^{1}$
}

Postoperative complications in esophagectomy for cancer. Evaluation of 215 cases according to definitions of the international consensus group

Introduction: Esophagectomy presents a high postoperative morbidity. However, the definitions used are variable. A multinational group (esophagectomy complications consensus group; ECCG) proposed standardized definitions. Aim: To evaluate postoperative complications in esophagectomy according to the definitions proposed by the ECCG. Materials and Method: We conducted a retrospective cohort study, based on a prospective database. Patients undergoing esophagectomy for cancer between 1996 and 2018 at one center were included. The definitions of postoperative complications according to the ECCG were applied. Results: We included 215 patients (64\% men, age 67 [31-82] years). Sixty-four percent had some comorbidity. There was a predominance of squamous carcinoma with $68 \%$. The most frequent tumor location was the lower third of the esophagus (48\%). An open approach was used in $74 \%$ and minimally invasive in $26 \%$. Total postoperative morbidity was $67 \%$. The most frequent complications were respiratory complications, which reached $27 \%$. The leakage of the esophagogastric anastomosis reached $25 \%, 24 \%$ were type II (did not require surgical reoperation). There was a vocal cord paresis in $7 \%$, all were classified as type I (did not require specific therapy). A chylous fistula was presented in $2 \%$, in $1 \%$ they were treated with parenteral nutrition (type II) and in $1 \%$ a reoperation was performed (type III). Conclusion: Esophagectomy is associated with a high morbidity. The main complications are respiratory and gastrointestinal. The use of consensus definitions allows standardization and grading of complications. Key words: esophageal neoplasms; esophageal cancer; esophagectomy; omplications consensus.

\section{Resumen}

Introducción: La esofagectomía presenta una alta morbilidad postoperatoria. Sin embargo, las definiciones de las complicaciones son variables. Un grupo multinacional (esophagectomy complications consensus group; ECCG) propuso definiciones estandarizadas. Objetivo: Evaluar las complicaciones postoperatorias en esofagectomía según las definiciones propuestas por el ECCG. Materiales y Método: Realizamos un estudio de cohorte retrospectivo, a partir de una base de datos prospectiva. Se incluyeron pacientes sometidos a una esofagectomía por cáncer entre 1996 y 2018 en un centro. Se aplicaron las definiciones de las complicaciones postoperatorias según el ECCG. Resultados: Se incluyeron 215 pacientes (Hombres 64\%; edad 67 [31-82] años). Un 64\% presentaban alguna comorbilidad. Existió un predominio de carcinoma escamoso con un $68 \%$. La ubicación tumoral más frecuente fue el tercio inferior del esófago (48\%). Se utilizó un abordaje abierto en 74\% y mínimamente invasivo en $26 \%$. La morbilidad postoperatoria total fue de $67 \%$. Las complicaciones más frecuentes fueron las respiratorias alcanzando un $27 \%$. En total, un $25 \%$ de los pacientes presentó una filtración de la anastomosis esofagogástrica, de las cuales un $24 \%$ fueron tipo II (no requirieron una reintervención quirúrgica). Se produjo una paresia de cuerda vocal en 7\%, todas tipo I (no requirieron terapia específica). Se presentó una fístula quilosa en $2 \%$, en $1 \%$ se trataron con nutrición parenteral (tipo II) y en 1\% se realizó una reintervención (tipo III). Conclusión: La esofagectomía se asocia a una alta morbilidad. Las principales complicaciones son las respiratorias y las gastrointestinales. La utilización de las definiciones de consenso permite una estandarización y graduación de las complicaciones. Palabras clave: neoplasias esofágicas; cáncer esofágico; esofagectomía; consenso de complicaciones.
${ }^{1}$ Hospital Dr. Sótero del Río. Equipo de Cirugía Esofagogástrica Departamento de Cirugía Digestiva. Pontificia Universidad Católica de Chile. Santiago, Chile.

Recibido el 9 de julio de 2019 y aceptado para publicación el 27 de octubre de 2019

Correspondencia a: Dr. Enrique Norero M. enorero@uc.cl enorero@yahoo.com 


\section{Introducción}

La esofagectomía es un pilar fundamental en el tratamiento del cáncer de esófago. Esta cirugía se asocia a una de las más altas mortalidades y morbilidades postoperatorias entre procedimientos electivos ${ }^{1-3}$. Las complicaciones influyen en el riesgo de mortalidad postoperatoria, en el tiempo de estadía hospitalaria, costos, reingreso, calidad de vida y pueden tener un efecto sobre la sobrevida a largo plazo ${ }^{4,5}$.

Existe una importante variación en la definición de las complicaciones postoperatorias entre distintos centros y publicaciones. Esto ha impedido una adecuada comparación entre distintas series y ha dificultado la evaluación y posterior mejoría de los resultados postoperatorios ${ }^{4,6}$.

Recientemente un grupo multinacional (esophagectomy complications consensus group; ECCG) compuesto por 21 cirujanos con amplia experiencia en cirugía esofágica y 14 países, propuso definiciones estandarizadas de complicaciones postoperatorias en esofagectomía ${ }^{4}$.

En nuestro país el cáncer esofágico presenta una baja prevalencia y se realizan un número reducido de esofagectomías al año ${ }^{7}$, lo cual también dificulta la estandarización en la definición de complicaciones postoperatorias.

El objetivo del estudio fue evaluar las complicaciones postoperatorias según las definiciones propuestas por el ECCG en un centro que tiene un registro prospectivo de esta cirugía.

\section{Materiales y Método}

Realizamos un estudio de cohorte retrospectiva, a partir de una base de datos prospectiva. Se incluyeron todos los pacientes sometidos a una esofagectomía por cáncer esofágico y de unión gastroesofágica de forma consecutiva entre 1996 y 2018 en un centro.

La evaluación preoperatoria del paciente consistió en hemograma, perfil bioquímico, pruebas hepáticas y ECG. Se realizó una evaluación nutricional. Se evaluó el riesgo cardiológico con un ECG, test de esfuerzo o cintigrama miocárdico y la función pulmonar mediante espirometría y gases arteriales. Desde 2013 los pacientes son evaluados preoperatoriamente por kinesiólogos y se someten a un programa de entrenamiento preoperatorio.

La evaluación del tumor consistió en una endoscopia y biopsia. En todos los pacientes se realizó un TC y/o PET-CT desde 2012. Se utilizó endosono- grafía para evaluar el T y N. En tumores de tercio medio y superior se realizó fibrobroncoscopía. Se utilizó el TNM según la séptima edición ${ }^{8}$.

Se indicó tratamiento neoadyuvante en pacientes con buena función cardiológica, renal y hematológica, que contaban con una vía de alimentación apropiada, clínicamente T3 o con ganglios positivos. Se realizó preferentemente radioterapia-quimioterapia en carcinoma escamoso y quimioterapia para casos de adenocarcinoma ${ }^{9,10}$.

\section{Técnica quirúrgica}

En la preparación anestésica se emplea un tubo orotraqueal de doble lumen en las esofagectomías realizadas por vía transtorácica y se utiliza analgesia mediante un catéter peridural.

La técnica quirúrgica se decidió según las características del paciente y el tumor.

Se realizó esofagectomía transhiatal abierta en pacientes con tumores de tercio inferior del esófago o tumores pequeños de los dos tercios superiores y en pacientes con mayores comorbilidades. Se utilizó una incisión media supraumbilical y cervical lateral izquierda, se disecó el hiato esofágico, con una maniobra de Pinotti. Posteriormente, se liberó el esófago de las estructuras del mediastino en forma roma y se realizó la linfadenectomía del mediastino inferior y abdominal.

Utilizamos una esofagectomía transtorácica abierta en tumores de los dos tercios superiores del esófago y en tumores de mayor tamaño. Se posicionó al paciente en decúbito lateral izquierdo. Se realiza una toracotomía posterolateral derecha en el $5^{\circ}$ espacio intercostal. Se secciona la vena ácigos. Se diseca el esófago y se realiza una linfadenectomía en bloque con la pieza incluyendo los ganglios infracarinales, paraesofágicos, supradiafragmáticos y del mediastino posterior. Una vez terminado el tiempo torácico, se cambia la posición del paciente a decúbito supino y se completa la linfadenectomía abdominal.

Desde el 2012 utilizamos en forma mayoritaria una esofagectomía mínimamente invasiva ${ }^{11}$, con una posición semiprono. Se instalan 4 trocares en el hemitórax derecho. Se diseca hacia superior, separando el esófago de la columna dorsal y de la vía aérea; $\mathrm{y}$ hacia el mediastino inferior separándolo del pericardio y aorta. Se incluye en la disección los ganglios periesofágicos e infracarinales. Se realiza una laparoscopia con 5-6 trocares en el abdomen superior. Se disecan los pilares y el tronco celiaco y sus ramas. Se realiza la linfadenectomía abdominal.

Para la reconstrucción se utiliza de preferencia el estómago, manteniendo la irrigación a través de 
los vasos gastroepiploicos derechos. Se asciende el estómago a través del mediastino posterior. Se realizó de rutina una yeyunostomía. No realizamos piloroplastía. En casos de tumores extensos de la unión gastroesofágica o en pacientes en que no se puede utilizar el estómago para la reconstrucción, realizamos una interposición de colon transverso ${ }^{12}$.

El manejo postoperatorio incluyó estadía en la unidad de recuperación y/o UCI y ventilación mecánica postoperatoria en forma selectiva. Se favorece la deambulación precoz y la kinesioterapia postoperatoria. Se mantiene la SNG por 5-7 días y se inicia alimentación por la yeyunostomía desde el tercer día postoperatorio. Se realimenta al paciente una vez retirada la SNG y se avanza a un régimen papilla antes del alta.

Se utilizaron las definiciones de complicaciones publicadas por el consenso internacional de esofagectomía ${ }^{4}$ y se graduó las complicaciones según la clasificación de Clavien ${ }^{13}$. Se evaluó la mortalidad postoperatoria a 30 días.

Esta clasificación define neumonía según la definición de la ATS IDSA y considera derrame pleural y neumotórax cuando requiere tratamiento o drenaje adicional. Se define falla respiratoria al requerir reintubación. Esta clasificación considera paresia de cuerdas vocales cuando se confirmó con nasofibroscopía ${ }^{4}$.

Se definió filtración de la anastomosis esófagogástrica como un defecto gastrointestinal independiente de la forma de presentación y del método utilizado en el diagnóstico (saliva por la incisión cervical, prueba de azul de metileno, amilasa del drenaje elevada, filtración por imágenes o en una reexploración quirúrgica). Se consideró retención gástrica como la necesidad de mantener la sonda nasogástrica pasado el día 7 postoperatorio ${ }^{4}$.

La fístula anastomótica, la necrosis gástrica, la paresia de las cuerdas vocales y la fístula quilosa se clasifican del I al III dependiendo del tratamiento que se aplica a cada una de estas complicaciones, donde la tipo III representa la necesidad de una nueva intervención quirúrgica ${ }^{4}$.

El comité de ética aprobó la realización del estudio. Se utilizó test de chi-cuadrado o Fisher para análisis estadístico.

\section{Resultados}

Se incluyeron 215 pacientes sometidos a una esofagectomía (hombres 64\%; edad 67 [31-82] años). Un $64 \%$ presentaba alguna comorbilidad, dentro de estos un 10\% se consideraron ASA III (Tabla 1).

Existió un predominio de carcinoma escamoso, con un $68 \%$ de casos con esta histología. La ubicación tumoral más frecuente fue el tercio inferior del esófago en 103 pacientes (48\%) (Tabla 1). En 36 (17\%) pacientes se realizó neoadyuvancia; en 22 con radioquimioterapia y en 14 con quimioterapia.

La mayoría de las esofagectomías realizadas fueron de carácter electivo $(98 \%)$ y en $5(2,8 \%)$ casos esta se realizó de urgencia, en 4 casos debido a una perforación esofágica. Se utilizó un abordaje abierto en $160(74 \%)$ casos y mínimamente invasivo en 55 $(26 \%)$. Se realizó una esofagectomía transhiatal abierta en 87 pacientes (40\%) y 24 pacientes $(11 \%)$ se sometieron a una esofagogastrectomía total transhiatal con interposición de colon. La esofagectomía transtorácica tipo Mckeown abierta se realizó en 48 casos $(22 \%)$. En todos los 55 casos de esofagectomía mínimamente invasiva se realizó un abordaje transtorácico. En 31 pacientes se realizó el tiempo abdominal por vía laparoscópica (Tabla 2).

La cirugía se consideró R0 en 168 (78\%), R1 en $16(7 \%)$ pacientes en su mayoría por compromiso del margen radial, R2 localmente en el mediastino en $18(8 \%)$ y $\mathrm{R} 2$ a distancia por compromiso metastásico en $13(6 \%)$ casos.

Tabla 1. Características preoperatorias de los pacientes y del tumor esofágico

\begin{tabular}{|lr|}
\hline N Sexo masculino & 215 \\
Edad (años) & $138(64 \%)$ \\
IMC $\left(\mathrm{kg} / \mathrm{mt}^{2}\right)$ & $67(31-82)$ \\
Comorbilidades & $23(14-37)$ \\
$\quad$ Hipertensión & \\
$\quad$ Diabetes & $76(35 \%)$ \\
Cardiopatía & $23(10 \%)$ \\
EPOC & $13(6 \%)$ \\
DHC & $9(4 \%)$ \\
Puntaje de ASA & $4(2 \%)$ \\
I & $77(36 \%)$ \\
II & $116(54 \%)$ \\
III & $22(10 \%)$ \\
Histología & \\
Carcinoma escamoso & $147(68 \%)$ \\
Adenocarcinoma & $62(29 \%)$ \\
Otros & $6(3 \%)$ \\
\hline Ubicación del tumor & \\
Tercio superior & $37(17 \%)$ \\
Tercio medio & $74(34 \%)$ \\
Tercio inferior & $103(48 \%)$ \\
\hline
\end{tabular}

IMC: Índice de masa corporal. EPOC: Enfermedad pulmonar obstructiva crónica. DHC: Daño hepático crónico. 
Para la reconstrucción del transitó se utilizó estómago en un $84 \%$ de los casos. No se realizó la reconstrucción en forma inmediata en un $3 \%$, en su mayoría debido a una esofagectomía de urgencia (Tabla 2).

Se presentaron complicaciones intraoperatorias en $12(6 \%)$ esofagectomías, la principal fue sangrado que requirió un cambio del abordaje con necesidad de toracotomía no programada o politransfusión en $5(2 \%)$ pacientes (Tabla 3$)$.

La morbilidad postoperatoria fue de $67 \%$ y se describe en detalle en la Tabla 4. En $56(26 \%)$ casos se produjeron complicaciones Clavien 3 o mayor. Las complicaciones más frecuentes fueron las respiratorias, que alcanzaron un $27 \%$. Y la filtración de la anastomosis esofagogástrica que llegó a 25\%.

Tabla 2. Técnica quirúrgica de esofagectomía

\begin{tabular}{|lr|}
\hline N & 215 \\
Técnica de esofagectomía & \\
Abierta & $160(74 \%)$ \\
Transhiatal & $87(40 \%)$ \\
Transtorácica & $49(23 \%)$ \\
McKeown & $48(22 \%)$ \\
Ivor Lewis & $1(0,5 \%)$ \\
Esofagogastrectomía total transhiatal & $24(11 \%)$ \\
Mínimamente invasiva & $55(26 \%)$ \\
Transhiatal & $0(0 \%)$ \\
Transtorácica & $55(26 \%)$ \\
McKeown & $45(21 \%)$ \\
Ivor Lewis & $10(5 \%)$ \\
Órgano de reconstrucción & $180(84 \%)$ \\
Estómago & $28(13 \%)$ \\
Colon &
\end{tabular}

Tabla 3. Complicaciones intraoperatorias anestésicas y quirúrgicas

\begin{tabular}{|ll|}
\hline Anestesia & \\
Lesión de vía aérea por tubo doble lumen & $1(0,5 \%)$ \\
Resección & \\
$\quad$ Sangrado & $5(2 \%)^{*}$ \\
Lesión de vía aérea & $1(0,5 \%)$ \\
Lesión de arco de vena ácigos & $1(0,5 \%)^{*}$ \\
Reconstrucción & \\
Esplenectomía necesidad & $1(0,5 \%)$ \\
Lesión de cola de páncreas & $1(0,5 \%)$ \\
Lesión vasos gastroepiploicos derechos & $2(1 \%)$ \\
\hline
\end{tabular}

*Sangrado 4 casos en disección transhiatal y 1 caso en disección transtorácica a nivel de arco aórtico, que obligó a conversión de la técnica o politransfusión.
Tabla 4. Complicaciones postoperatorias en esofagectomía

\begin{tabular}{|c|c|}
\hline Respiratorias & $59(27 \%)$ \\
\hline Neumonía & $44(20 \%)$ \\
\hline Derrame pleural & $14(7 \%)$ \\
\hline Neumotórax & $7(3 \%)$ \\
\hline Falla respiratoria (ventilación mecánica) & $20(9 \%)$ \\
\hline Aspiración & $1(0,4 \%)$ \\
\hline Lesión traqueobronquial & $3(1 \%)$ \\
\hline \multicolumn{2}{|l|}{ Cardíacas } \\
\hline Paro cardiorrespiratorio & $1(0,4 \%)$ \\
\hline Disrritmia atrial & $15(7 \%)$ \\
\hline Falla cardíaca congestiva & $3(1 \%)$ \\
\hline \multicolumn{2}{|l|}{ Gastrointestinales } \\
\hline Fístula anastomosis esofagogástrica & $52(25 \%)$ \\
\hline Tipo I & $0(0 \%)$ \\
\hline Tipo II & $49(24 \%)$ \\
\hline Tipo III & $3(1 \%)$ \\
\hline Necrosis tubo gástrico & $6(3 \%)$ \\
\hline Tipo I-II & $2(1 \%)$ \\
\hline Tipo IIIi & $4(2 \%)$ \\
\hline Obstrucción intestinal & $1(0,4 \%)$ \\
\hline Complicaciones yeyunostomía & $4(2 \%)$ \\
\hline Clostridium difficile & $7(3 \%)$ \\
\hline Vaciamiento gástrico enlentecido & $24(13 \%)$ \\
\hline \multicolumn{2}{|l|}{ Urológicas } \\
\hline Falla renal aguda & $8(4 \%)$ \\
\hline Infección urinaria & $7(3 \%)$ \\
\hline \multicolumn{2}{|l|}{ Tromboembólicas } \\
\hline Tromboembolismo pulmonar & $5(2 \%)$ \\
\hline \multicolumn{2}{|l|}{ Neurológicas/psiquiátricas } \\
\hline Paresia de cuerda vocal & $14(7 \%)$ \\
\hline Tipo I & $14(7 \%)$ \\
\hline Tipo II-III & $0(0 \%)$ \\
\hline A. Unilateral & $11(5 \%)$ \\
\hline B. Bilateral & $3(1 \%)$ \\
\hline Delirio & $8(4 \%)$ \\
\hline \multicolumn{2}{|l|}{ Infecciosas } \\
\hline Infección de la herida & $10(5 \%)$ \\
\hline Infección catéter venoso central & $6(2,8 \%)$ \\
\hline Absceso abdominal-torácico & $6(2,8 \%)$ \\
\hline \multicolumn{2}{|l|}{ Herida y diafragma } \\
\hline Evisceración & $2(1 \%)$ \\
\hline \multicolumn{2}{|l|}{ Otros } \\
\hline Fístula quilosa & $4(2 \%)$ \\
\hline Tipo I & $0(0 \%)$ \\
\hline Tipo II & $2(1 \%)$ \\
\hline Tipo III & $2(1 \%)$ \\
\hline \multicolumn{2}{|l|}{ Reoperaciones por otros motivos } \\
\hline Filtración abdominal & 3 \\
\hline Lesión intestinal & 2 \\
\hline Morbilidad postoperatoria & $144(67 \%)$ \\
\hline
\end{tabular}


Entre las complicaciones respiratorias destaca la neumonía en un $20 \%$ de casos. En segundo lugar la falla respiratoria que requirió ventilación mecánica en un $9 \%$. La necesidad de procedimientos para tratar derrames pleurales o neumotórax fue de $7 \%$ y $3 \%$ respectivamente.

De las complicaciones cardiacas, la más frecuente fue la arritmia atrial en un $7 \%$, en su mayoría fibrilación auricular.

En las complicaciones gastrointestinales se produjeron 49 filtraciones de anastomosis cervical y 3 filtraciones en anastomosis torácicas. La fístula de la anastomosis esofagogástrica se clasificó como tipo II en $24 \%$, es decir, que se manejó con apertura de la herida cervical y antibióticos. En 3 (1\%) casos se clasificó como tipo III, es decir, requirió una reintervención quirúrgica; un caso de aseo en pabellón de una anastomosis cervical; en otro caso se desmanteló una anastomosis cervical debido a filtración descendente hacia el mediastino y cavidad pleural derecha; y en un tercer caso se realizó un aseo por videotoracoscopia debido a una filtración de una anastomosis torácica.

En 6 casos (3\%) se produjo una filtración y/o necrosis del estómago ascendido en la reconstrucción. En 4 (2\%) casos se clasificó como tipo III, es decir, que requirió una resección del estómago ascendido.

La tercera complicación gastrointestinal identificada fue el vaciamiento gástrico enlentecido, que se produjo en un $13 \%$.

Entre las complicaciones neurológicas destaca la paresia de las cuerdas vocales en un 7\%, que se clasificó como tipo I en todos los casos, es decir, que no se realiza ningún procedimiento quirúrgico sobre las cuerdas vocales para tratarlo.

Se produjo una fístula quilosa en un $2 \%$ de casos. En 2 casos se manejó con nutrición parenteral (tipo II) y en 2 casos se decidió una reintervención para ligar el conducto torácico por videotoracoscopia (tipo III).

La mortalidad operatoria a 30 días fue de $7,4 \%$. Existió una disminución significativa de la mortalidad postoperatoria al comparar el periodo 19962012 y el período 2013-2018. Previo al 2013 la mortalidad a 30 días fue de $9 \%$ y disminuyó a 1,7\% entre los años 2013 y $2018(p=0,04)$. La mediana de estadía postoperatoria fue de 18 días.

\section{Discusión}

La esofagectomía es uno de los procedimientos con más alta morbilidad postoperatoria en cirugía oncológica digestiva ${ }^{1,2}$. Los datos presentados en este trabajo apoyan esto, demostrándose un $67 \%$ de morbilidad postoperatoria.

Sin embargo, existe una gran disparidad en la descripción de las complicaciones en distintas series e instituciones ${ }^{6,14-16}$. Esto en parte puede estar dado por las diferencias en la definición utilizada por los distintos centros ${ }^{6}$. Debido a esto en 2015 se publica el consenso internacional (Esophagectomy complications consensus group; ECCG) que propone definiciones estandarizadas de complicaciones postoperatorias en esofagectomía ${ }^{4}$. En nuestro estudio hemos utilizado estas definiciones y hemos identificado como principales complicaciones a las respiratorias y las gastrointestinales.

Por otro lado, este consenso busca graduar en gravedad algunas complicaciones que son específicas para esta operación; la fístula anastomótica, la necrosis gástrica, la paresia de las cuerdas vocales y la fistula quilosa ${ }^{4}$. Esta graduación específica de la severidad de las complicaciones apunta en la misma dirección que la más universal clasificación de Clavien $^{13}$, y también favorece la comparabilidad entre casuísticas ya que, por ejemplo en algunos casos, se comunica solo las fístulas graves que requieren una reintervención, pero no las menores que se manejan en forma conservadora ${ }^{14}$.

La filtración de la anastomosis esofagogástrica se presenta en la literatura con cifras muy variables, entre $2 \%$ y $21 \%{ }^{15,17,18}$. Sin embargo, pocos estudios han diferenciado fistulas de menor y mayor gravedad $^{19}$. Este nuevo sistema permite clasificarlas en tipo I, II y $\mathrm{III}^{4}$. En nuestro estudio demostramos que la minoría (1\%) de las filtraciones requieren una reintervención quirúrgica (tipo III) y la mayoría son tipo II, es decir, se manejan abriendo la incisión cervical y manejo local.

La necrosis del tubo gástrico se describe como una complicación en esofagectomía entre $2 \%$ y $10 \%$ de los $\operatorname{casos}^{18,20}$. El diagnóstico de esta complicación puede variar significativamente. En nuestros datos la identificamos como una complicación grave (tipo III) que se trató con una reintervención en 4 casos.

La paresia de las cuerdas vocales se describe entre un $3 \%$ a $9 \%{ }^{3}$. En este sistema se exige la confirmación de la paresia mediante examen directo ${ }^{4}$, lo cual no estaba previamente estandarizado en la literatura. En nuestro estudio, se produjo en un 7\% de casos y en todos los casos esta se clasificó como tipo I, es decir, que no requirió una reintervención sobre las cuerdas vocales.

La fístula quilosa se describe entre un $1 \% \mathrm{y}$ $5 \%{ }^{18,21}$ en la literatura. En nuestros datos se produjo en un $2 \%$ y requirió una reexploración para ligar el conducto torácico en la mitad de los casos. Esta es 
una complicación rara, pero grave, ya que lleva a la rápida desnutrición del paciente. $\mathrm{Y}$ es una complicación en gran medida evitable realizando una adecuada ligadura del ducto torácico en la cirugía inicial ${ }^{22}$.

Al comparar los resultados de complicaciones en esta publicación con los resultados de nuestro mismo grupo presentados en el Congreso Chileno de Cirugía en 2014 sobre esofagectomía en 178 pacientes entre 1996 a 2014, encontramos una disminución de las complicaciones globales (previamente $74 \%$ y actualmente $67 \%$ ) y en las complicaciones respiratorias (previamente $35 \%$ y actualmente $27 \%$ ). Los motivos de esta disminución son difíciles de evaluar, pero es posible que una definición más precisa en las complicaciones respiratorias, el uso preferencial del abordaje mínimamente invasivo y una mejor atención kinésica pueden explicar este cambio.

Las complicaciones respiratorias son frecuentes en esofagectomía llegando a $30 \%$ en la literatura ${ }^{3,18}$. En nuestro estudio se produjeron estas complicaciones en un $27 \%$. Estas complicaciones son multifactoriales dependiendo de las comorbilidades, el antecedente de tabaquismo, el uso de radioterapia preoperatoria, abordaje transtorácico abierto y manejo insuficiente del dolor postoperatorio ${ }^{3,23}$. En la literatura se describe que la prehabilitación de estos pacientes puede mejorar estos resultados ${ }^{24}$, por lo cual hemos iniciado un protocolo de intervención por kinesioterapia tanto pre como postoperatorio. $\mathrm{Y}$ juntos con esto también hemos cambiado el abordaje abierto por uno mínimamente invasivo ${ }^{11}$, lo cual según la literatura disminuye las infecciones respiratorias ${ }^{25}$.

Una limitación del estudio es que si bien se to- maron los datos de una base de datos prospectiva, la aplicación de las definiciones de complicaciones se realizó en forma retrospectiva. En segundo lugar, este estudio cubre 22 años de duración y se han realizado cambios significativos al manejo de estos pacientes, por lo que es posible que las complicaciones hayan cambiado a lo largo del tiempo. Y por último no fue posible tener datos sobre otros aspectos que se describen en el consenso internacional tales como transfusión de glóbulos rojos, reingresos y resultados a 90 días.

\section{Conclusión}

La esofagectomía es una cirugía con alta morbilidad postoperatoria. Las principales complicaciones son las respiratorias y las gastrointestinales. La utilización de un sistema estandarizado de evaluación de complicaciones permite una comparación adecuada con otras series y representa una base para buscar mejorar los resultados postoperatorios.

\section{Responsabilidades éticas}

Protección de personas y animales. Los autores declaran que para esta investigación no se han realizado experimentos en seres humanos ni en animales.

Confidencialidad de los datos. Los autores declaran que en este artículo no aparecen datos de pacientes.

Conflictos de interés: no hay.

\section{Bibliografía}

1. Ghaferi AA, Birkmeyer JD, Dimick JB. Complications, failure to rescue, and mortality with major inpatient surgery in medicare patients. Ann Surg. 2009;250:1029-34.

2. Birkmeyer JD, Siewers AE, Finlayson EV, Stukel TA, Lucas FL, Batista I, et al. Hospital volume and surgical mortality in the United States. N Engl J Med. 2002;346:1128-37.

3. Braghetto I, Csendes A, Cardemil G, Burdiles P, Rodríguez A, Guerra JF. Complicaciones de la esofagectomía: diagnóstico, mecanismo fisiopatológico, prevención y manejo. Rev Chil Cir. 2002;54:451-63.
4. Low DE, Alderson D, Cecconello I, Chang AC, Darling GE, D'Journo $\mathrm{XB}$, et al. International Consensus on Standardization of Data Collection for Complications Associated With Esophagectomy: Esophagectomy Complications Consensus Group (ECCG). Ann Surg. 2015;262:286-94.

5. Lerut T, Moons J, Coosemans W, Van Raemdonck D, De Leyn P, Decaluwé H, et al. Postoperative complications after transthoracic esophagectomy for cancer of the esophagus and gastroesophageal junction are correlated with early cancer recurrence: role of systematic grading of complications using the modified Clavien classification. Ann Surg. 2009;250:798807.
6. Blencowe NS, Strong S, McNair AG, Brookes ST, Crosby T, Griffin SM, et al. Reporting of short-term clinical outcomes after esophagectomy: a systematic review. Ann Surg. 2012;255:658-66.

7. Csendes A, González G. Cirugías digestivas más frecuentes en Chile, excluyendo colorrectal. Rev Chil Cir. 2008;60:379-86.

8. Talsma K, van Hagen P, Grotenhuis BA, Steyerberg EW, Tilanus HW, van Lanschot JJ, et al. Comparison of the 6th and 7th Editions of the UICC-AJCC TNM Classification for Esophageal Cancer. Ann Surg Oncol. 2012;19:2142-8.

9. van Hagen P, Hulshof MC, van Lanschot JJ, Steyerberg EW, van Berge Henegouwen MI, Wijnhoven BP, et al. 
Preoperative chemoradiotherapy for esophageal or junctional cancer. N Engl J Med. 2012;366:2074-84.

10. Ychou M, Boige V, Pignon JP, Conroy T, Bouché O, Lebreton G, et al. Perioperative chemotherapy compared with surgery alone for resectable gastroesophageal adenocarcinoma: an FNCLCC and FFCD multicenter phase III trial. J Clin Oncol. 2011;29:1715-21.

11. Norero E, Ceroni M, Ramírez A, Mejía R, Martínez C, Muñoz R, et al. Experiencia inicial con esofagectomía mínimamente invasiva transtorácica en posición semiprono por cáncer esofágico. Rev Chil Cir. 2018;70:27-34.

12. Ceroni M, Norero E, Henríquez JP, Viñuela E, Briceño E, Martínez C, et al. Total esophagogastrectomy plus extended lymphadenectomy with transverse colon interposition: A treatment for extensive esophagogastric junction cancer. World J Hepatol. 2015;7:2411-7.

13. Dindo D, Demartines N, Clavien PA. Classification of surgical complications: a new proposal with evaluation in a cohort of 6336 patients and results of a survey. Ann Surg. 2004;240:205-13.

14. Luketich JD, Pennathur A, Awais O, Levy RM, Keeley S, Shende M, et al. Outcomes after minimally invasive esophagectomy: review of over 1000 patients. Ann Surg. 2012;256:95-103.

15. Palanivelu C, Prakash A, Senthilkumar R, Senthilnathan P, Parthasarathi R, Rajan PS, et al. Minimally invasive esophagectomy: thoracoscopic mobilization of the esophagus and mediastinal lymphadenectomy in prone position--experience of 130 patients. J Am Coll Surg. 2006;203:7-16.

16. Swanson SJ, Batirel HF, Bueno R, Jaklitsch MT, Lukanich JM, Allred E, et al. Transthoracic esophagectomy with radical mediastinal and abdominal lymph node dissection and cervical esophagogastrostomy for esophageal carcinoma. Ann Thorac Surg. 2001;72:1918-24; discussion 24-5.

17. van Rossum PS, Haverkamp L, Carvello M, Ruurda JP, van Hillegersberg R. Management and outcome of cervical versus intrathoracic manifestation of cervical anastomotic leakage after transthoracic esophagectomy for cancer. Dis Esophagus 2017;30:1-8.

18. Schmidt HM, Gisbertz SS, Moons J, Rouvelas I, Kauppi J, Brown A, et al. Defining Benchmarks for Transthoracic Esophagectomy: A Multicenter Analysis of Total Minimally Invasive Esophagectomy in Low Risk Patients. Ann Surg. 2017;266:814-21.

19. Zehetner J, DeMeester SR, Alicuben ET, Oh DS, Lipham JC, Hagen JA, et al. Intraoperative Assessment of Perfusion of the Gastric Graft and Correlation With Anastomotic Leaks After Esophagectomy. Ann Surg. 2015;262:74-8.

20. Veeramootoo D, Parameswaran R, Krishnadas R, Froeschle P, Cooper M, Berrisford RG, et al. Classification and early recognition of gastric conduit failure after minimally invasive esophagectomy. Surg Endosc. 2009;23:2110-6.

21. Braghetto I, Csendes A, Cornejo A, Amat J, Cardemil G, Burdiles P, et al. Survival of patients with esophageal cancer subjected to total thoracic esophagectomy. Rev Med Chile 2000;128:64-74.

22. Guo W, Zhao YP, Jiang YG, Niu HJ, Liu XH, Ma Z, et al. Prevention of postoperative chylothorax with thoracic duct ligation during video-assisted thoracoscopic esophagectomy for cancer. Surg Endosc. 2012;26:1332-6.

23. Minnella EM, Awasthi R, Loiselle SE, Agnihotram RV, Ferri LE, Carli F. Effect of Exercise and Nutrition Prehabilitation on Functional Capacity in Esophagogastric Cancer Surgery: A Randomized Clinical Trial. JAMA Surg. 2018 (Epub ahead of print).

24. Christensen JF, Simonsen C, BanckPetersen A, Thorsen-Streit S, Herrstedt A, Djurhuus SS, et al. Safety and feasibility of preoperative exercise training during neoadjuvant treatment before surgery for adenocarcinoma of the gastro-oesophageal junction. BJS Open. 2019;3:74-84.

25. Biere SS, van Berge Henegouwen MI, Maas KW, Bonavina L, Rosman C, García $\mathrm{JR}$, et al. Minimally invasive versus open oesophagectomy for patients with oesophageal cancer: a multicentre, openlabel, randomised controlled trial. Lancet. 2012;379(9829):1887-92. 